\title{
Effective ICP Reduction by Decompressive Craniectomy in Patients with Severe Traumatic Brain Injury Treated by an ICP-Targeted Therapy
}

\author{
MAGNUS OLIVECRONA, ${ }^{1}$ MARIE RODLING-WAHLSTRÖM, ${ }^{2}$ SILVANA NAREDI, \\ and LARS-OWE D. KOSKINEN ${ }^{1}$
}

\begin{abstract}
Severe traumatic brain injury (TBI) is one of the major causes of death in younger age groups. In Umeå, Sweden, an intracranial pressure (ICP) targeted therapy protocol, the Lund concept, has been used in treatment of severe TBI since 1994. Decompressive craniectomy is used as a protocolguided treatment step. The primary aim of the investigation was to study the effect of craniectomy on ICP changes over time in patients with severe TBI treated by an ICP-targeted protocol. In this retrospective study, all patients treated for severe TBI during 1998-2001 who fulfilled the following inclusion criteria were studied: GCS $\leq 8$ at intubation and sedation, first recorded cerebral perfusion pressure (CPP) of $>10 \mathrm{~mm} \mathrm{Hg}$, arrival within $24 \mathrm{~h}$ of trauma, and need of intensive care for $>72 \mathrm{~h}$. Craniectomy was performed when the ICP could not be controlled by evacuation of hematomas, sedation, ventriculostomy, or low-dose pentothal infusion. Ninety-three patients met the inclusion criteria. Mean age was $\mathbf{3 7 . 6}$ years. Twenty-one patients underwent craniectomy as a treatment step. We found a significant reduction of the ICP directly after craniectomy, from $36.4 \mathrm{~mm}$ $\mathrm{Hg}$ (range, 18-80 $\mathrm{mm} \mathrm{Hg}$ ) to $12.6 \mathrm{~mm} \mathrm{Hg}$ (range, 2-51 mm Hg). During the following $72 \mathrm{~h}$, we observed an increase in ICP during the first 8-12 h after craniectomy, reaching approximately $20 \mathrm{~mm}$ $\mathrm{Hg}$, and later levelling out at approximately $25 \mathrm{~mm} \mathrm{Hg}$. The reduction of ICP was statistically significant during the $72 \mathrm{~h}$. The outcome as measured by Glasgow Outcome Scale (GOS) did not significantly differ between the craniectomized group (DC) and the non-craniectomized group (NDC). The outcome was favorable (GOS 5-4) in $71 \%$ in the craniectomized group, and in $61 \%$ in the noncraniectomized group. Craniectomy is a useful tool in achieving a significant reduction of ICP over time in TBI patients with progressive intracranial hypertension refractory to medical therapy. The procedure seems to have a satisfactory effect on the outcome, as demonstrated by a high rate of favorable outcome and low mortality in the craniectomized group, which did not significantly differ compared with the non-craniectomized group.
\end{abstract}

Key words: decompressive craniectomy; head injury; ICP; Lund concept; outcome

Departments of ${ }^{1}$ Neurosurgery and ${ }^{2}$ Anesthesiology, University Hospital, Umeå, Sweden. 


\section{OLIVECRONA ET AL.}

\section{INTRODUCTION}

QEVERE TRAumatic BRAIN InJURy (TBI) is a major cause of death and disability in the population, particularly in the younger age groups. There have been several strategies to decrease the mortality in TBI. The importance of management of intracranial pressure (ICP) in TBI has been understood for a long time. To reduce ICP during intracranial hypertension, decompressive craniectomy (DC) has been used (Kocher, 1901; Cushing, 1905). During the past decades, several different experiences with DC in TBI have been reported (Gaab et al., 1990; Polin et al., 1997; Guerra et al., 1999; Meier et al., 2000; Munch et al., 2000; Whitfield et al., 2001; Hejazi et al., 2002; Schneider et al., 2002; Albanese et al., 2003; Meier and Grawe, 2003).
In 1993, the so-called Lund concept for the treatment of TBI was introduced. Its aim is to reduce the ICP and improve the microcirculation in the areas surrounding severe brain contusions (Fig. 1). The physiological forces that regulate fluid fluxes across the injured blood-brain barrier are actively optimized in order to reduce the postinjury edema (Asgeirsson et al., 1994; Grande et al., 1997, 2002). This treatment concept was introduced in our clinical practice in 1994. Four outcome studies published from three different university hospitals in Sweden using the protocol have shown a mortality rate of about $10 \%$ and favorable outcomes as indicated by Glasgow Outcome Scale (GOS) 4-5 in $70 \%$ of the patients suffering from severe TBI (Eker et al., 1998; Naredi et al., 1998, 2001; Wahlstrom et al., 2005). In 1997, we introduced the use of decompressive hemi-craniectomy as

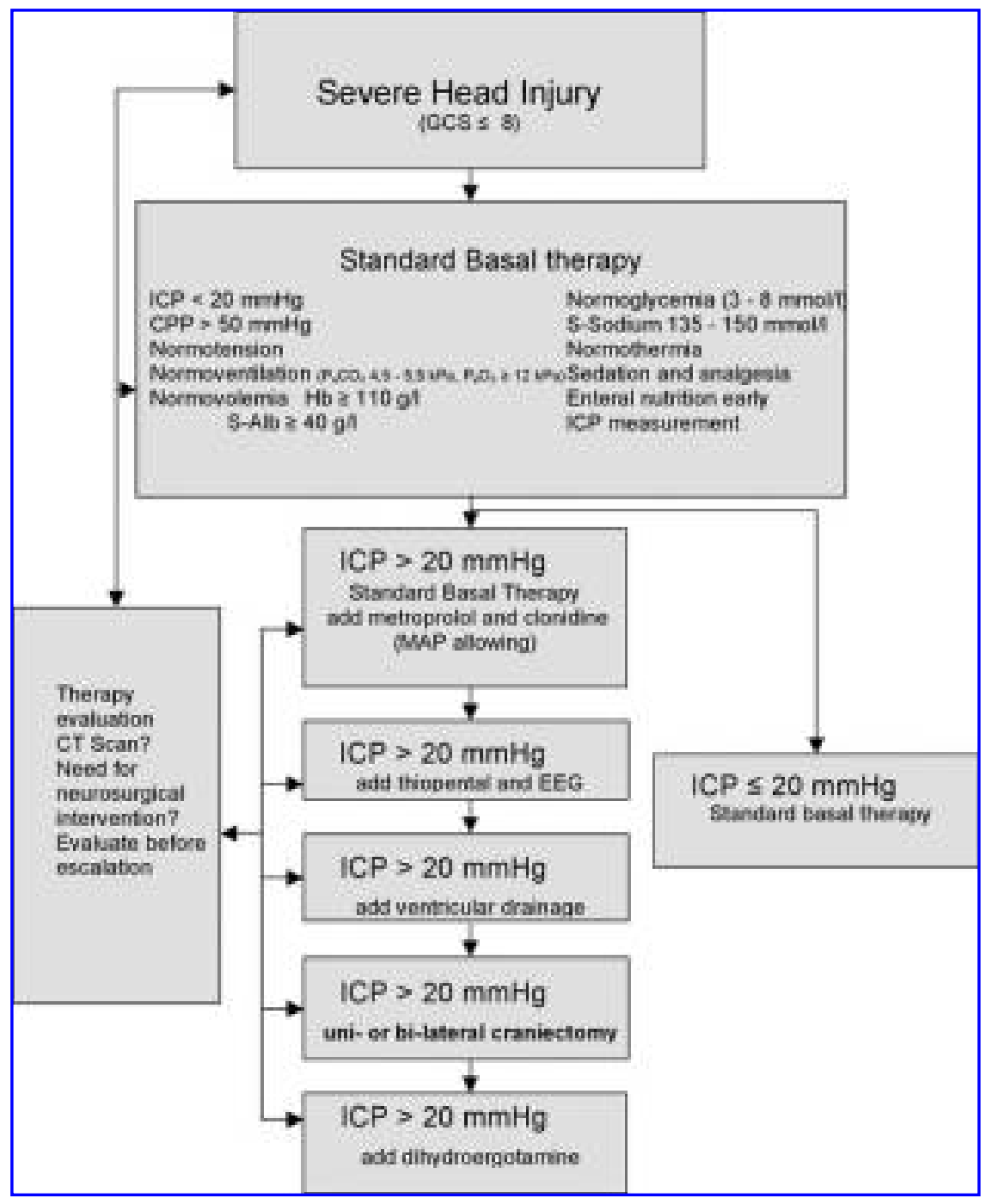

FIG. 1. Flow chart of the treatment protocol. 


\section{DECOMPRESSIVE CRANIECTOMY AND ICP REDUCTION}

an additional step in our protocol-guided treatment of TBI.

We hypothesized that DC has a beneficial effect in TBI patients treated by the Lund protocol who have refractory intracranial hypertension, and we tested this hypothesis in a retrospective analysis of consecutive patients with severe TBI treated at our department during 1998-2001 with the focus on the effect of the DC. The main aim of the study was to analyze the changes in ICP and cerebral perfusion pressure (CPP) over time in DC patients and to compare the outcome between DC patients and non-DC patients (NDC). Secondarily, we aimed to analyze if age and initial severity of trauma influenced the patient outcome.

\section{METHODS}

\section{Patient Material}

The Department of Neurosurgery at the Umeå University Hospital, in the northern part of Sweden, has a regional responsibility for a population of approximately 900,000 inhabitants, covering almost half the area of Sweden. All the referring local hospitals refer severe TBI patients to us, and during the period of investigation, no severe TBI was denied admittance to Umeå University Hospital, including patients with dilated fixed pupils, complicating illness or other injuries, and below the age of 75 years.

All patients treated for severe TBI at our hospital during the time period of January 1, 1998, to December 31, 2001, were identified. Those who were accepted for the study had a verified head injury, a level of consciousness of Glasgow Coma Scale (GCS) of 8 or worse at the time of sedation, and intubation, and they had a first recorded CPP of $10 \mathrm{~mm} \mathrm{Hg}$ or higher, irrespective of the ICP or the size or reactivity of the pupils. The patients should have arrived in our hospital not later than $24 \mathrm{~h}$ after the trauma. Those requiring intensive care for less than $72 \mathrm{~h}$ were excluded since patients who could leave the ICU alive within $72 \mathrm{~h}$ had head injuries that were regarded as not severe despite a GCS of $\leq 8$ at intubation and sedation. Patients who died within the first $72 \mathrm{~h}$ were included in the mortality group. Patients deceased during the first 3 months after injury irrespective of their ICU time (even if it was less than $72 \mathrm{~h}$ ) were classified as mortalities. Penetrating head injury was an exclusion criterion.

The study was approved by the local ethics committee of the Umeå University (dnr 00/175).

\section{Monitoring}

All patients received a parenchymal ICP measuring device (Codman MicroSensor ${ }^{\mathrm{TM}}$, Johnson \& Johnson Pro- fessional Inc., Raynham, MA) or ventriculostomy, and ICP was recorded continuously during the treatment period. The patients were initially treated in a supine position with no head elevation. The pre-auricular level was used as the zero-reference for ICP measurement by ventriculostomy. Arterial blood pressure was continuously measured in the radial artery with the right atrial level used as a zero-reference. Oxyhemoglobin percentage was recorded by pulse oximetry (Marquette Solar, General Electric Medical Systems, Milwaukee, WI). Further, oxygen saturation and end-tidal partial pressure carbon dioxide (Marquette Solar) were continuously recorded. Frequent blood gas analyses were performed, and all other basic blood and physiological parameters were followed. Central venous pressure was recorded, as well as electrocardiography (ECG). All the collected data were stored digitally in a computer.

The primary admitting hospital performed an initial computed tomography (CT) scan of the brain. This was often repeated upon arrival after transfer to our hospital. In accordance with the treatment protocol, a CT scan of the brain was performed approximately $24 \mathrm{~h}$ after trauma. Further CT investigations were performed if unexpected changes in the clinical status occurred.

\section{Treatment}

The treatment mostly followed the original guidelines for the Lund concept (Fig. 1). Patients were sedated with midazolam and fentanyl with the goal of achieving a level of comfortable sedation while still allowing them to cough. The level of sedation was monitored continuously or daily with electroencephalography (EEG). Intracranial mass lesions were aggressively treated surgically. Patients were kept on the ventilator, in a controlled breathing mode aimed at maintaining normal $\mathrm{pH}$ and a $\mathrm{P}_{\mathrm{a}} \mathrm{CO}_{2}$ of 4.5-5.5 kPa. Intravascular fluid therapy was guided by several simultaneous goals: adequate intravascular fluid volume, a daily negative total fluid balance, $\mathrm{Hb}$ level of $\geq 110 \mathrm{~g} / \mathrm{L}$, and serum albumin of $\geq 40 \mathrm{~g} / \mathrm{L}$. This was achieved by using combinations of infusion of packed red blood cells, albumin, glucose, Ringer's acetate, and furosemide. The following interventions were based on the level of ICP and its trend. If the ICP increased and remained above $20 \mathrm{~mm} \mathrm{Hg}$, a new CT scan was performed to rule out any lesion that could be surgically removed. If there was no mass lesion to evacuate and if the mean arterial pressure (MAP) allowed metoprolol and/or clonidine was infused intravenously in order to reduce the hydrostatic capillary pressure, to prevent further edema formation, allowing the CPP to be reduced towards but not below $50 \mathrm{~mm} \mathrm{Hg}$.

Further, these drugs reduce the general stress reactions mediated by the sympathetic nervous system. If the in- 


\section{OLIVECRONA ET AL.}

creased ICP did not respond to this intervention, a new CT scan was performed. If the CT scan ruled out the need for surgical intervention, the medical therapy was escalated using an infusion of sodium pentothal, 0.5-3 $\mathrm{mg} / \mathrm{kg} / \mathrm{h}$, aiming at achieving a "delta" pattern on the EEG. If the increased ICP did not respond to this treatment, a new CT scan was performed, and if no mass lesion suitable for surgical removal was identified, a ventriculostomy was placed. The ventricular drain was kept closed and only opened briefly to let out small amounts of cerebrospinal fluid (CSF) in order to lower the ICP. Care was taken not to completely drain the ventricles.

\section{Craniectomy}

If the ICP could not be controlled by these steps, either unilateral or bilateral craniectomy was indicated when an increasing trend in ICP was observed and the ICP further increased and rested at a level above $25 \mathrm{~mm}$ $\mathrm{Hg}$. Before proceeding to craniectomy, all steps in the medical therapy were re-evaluated, and the patient was confirmed to be optimally managed in accordance with the treatment protocol. Whether the craniectomy was performed unilaterally or bilaterally was decided based on the CT scan results. The craniectomy was performed on the side were the expansivity was most pronounced; however, in cases with general swelling, a bilateral craniectomy was performed. We used a "question mark"-shaped skin incision for unilateral craniectomy. For bilateral craniectomies, a bi-coronal skin incision was made and then supplemented by an incision going posteriorly in the midline. In most cases, a musculo-cutaneous skin flap was used. The craniectomy area was designed to cover at least $12 \times 8 \mathrm{~cm}$.

The dura was opened with radiating cuts, and a large duraplasty measuring at least $4 \times 10 \mathrm{~cm}$ was performed in order to allow the brain to expand into the space opened by the craniectomy. The dura itself is almost non-expansile, so no significant brain expansion volume gain will be achieved by craniectomy if a duraplasty is not performed. There was attention given to perform a large enough DC and duraplasty in order to gain as much brain expansion volume as possible. The area of the craniectomy was calculated from a post-operative CT scan using the simple method of an ellipsoid. The bulging of the brain was estimated to be $10 \%$ of the mean diameter of the craniectomy as measured on the post-craniectomy CT scan. This was used for the calculation of the volume gained by surgery.

If all medical and surgical interventions fail, dihydroergotamine is the last option in the treatment protocol, and can be used in order to lower the hydrostatic transcapillary pressure, thus reducing edema formation (Asgeirsson et al., 1994).

\section{Outcome}

The GOS was used for assessment of clinical status during the recovery phase. Two nurses and a physician, who were not investigators in the study, performed the GOS scoring. A letter was sent to the patients or relatives explaining that they would be interviewed by telephone. The structured interview followed the GOS guidelines for assessment.

\section{Statistical Analysis}

Results are reported as means \pm standard error of the mean (SEM) or, in cases of discrete variables, as median and range. Analysis of variance (ANOVA) followed by the post-hoc Fischer PLSD test was used for calculating statistically significant differences when several comparisons were made. For discrete variables, the Mann-Whitney $U$-test was employed, and the two-tailed Student's $t$ test for continuous variables was used when applicable.

\section{RESULTS}

Ninety-three patients fulfilled the criteria and were included in the study. There were 71 males and 22 females. The mean age at accident was $37.6 \pm 1.7$ years. DC was performed in $21(23 \%)$ of these patients, and this group (15 males, six females) had a mean age of $39.4 \pm 3.4$ years. DC was performed after an average of $45 \mathrm{~h}(2-157$ h) of mean treatment time in our intensive care unit (ICU; Fig. 2). The median GCS at intubation and sedation was 7 (range, 8-3) for the whole group and exactly the same in the DC group. The NDC group showed the same median GCS, i.e., 7 (range, 8-3). Thirteen patients showed a GCS of 3. For detailed information on number, gender, age, and GCS see Table 1. There was no difference between the DC and NDC groups in regard to age (Student's unpaired two-tailed $t$-test), gender, or GCS (MannWhitney $U$-test).

The mean area of the craniectomy was $88 \pm 7 \mathrm{~cm}^{2}$ in the unilateral cases and $116 \pm 11 \mathrm{~cm}^{2}$ when both unilateral and bilateral cases were included. The corresponding calculated volumes were $98 \pm 11$ and $124 \pm 14 \mathrm{~cm}^{3}$, respectively.

The mean daily ICP in the DC group was above 20 $\mathrm{mm} \mathrm{Hg}$ for the first 7 days. The difference between the DC and NDC groups in mean daily ICP was statistically significant $(p<0.001$, ANOVA). As demonstrated in Figure 3, the difference between the mean daily ICP in the two groups was statistically significant up to day 6 . Figure 3 also shows that the mean daily CPP in both the NDC group and in the DC group was above the lowest accepted CPP value, i.e., $50 \mathrm{~mm} \mathrm{Hg}$. Figure 4 shows the 


\section{DECOMPRESSIVE CRANIECTOMY AND ICP REDUCTION}

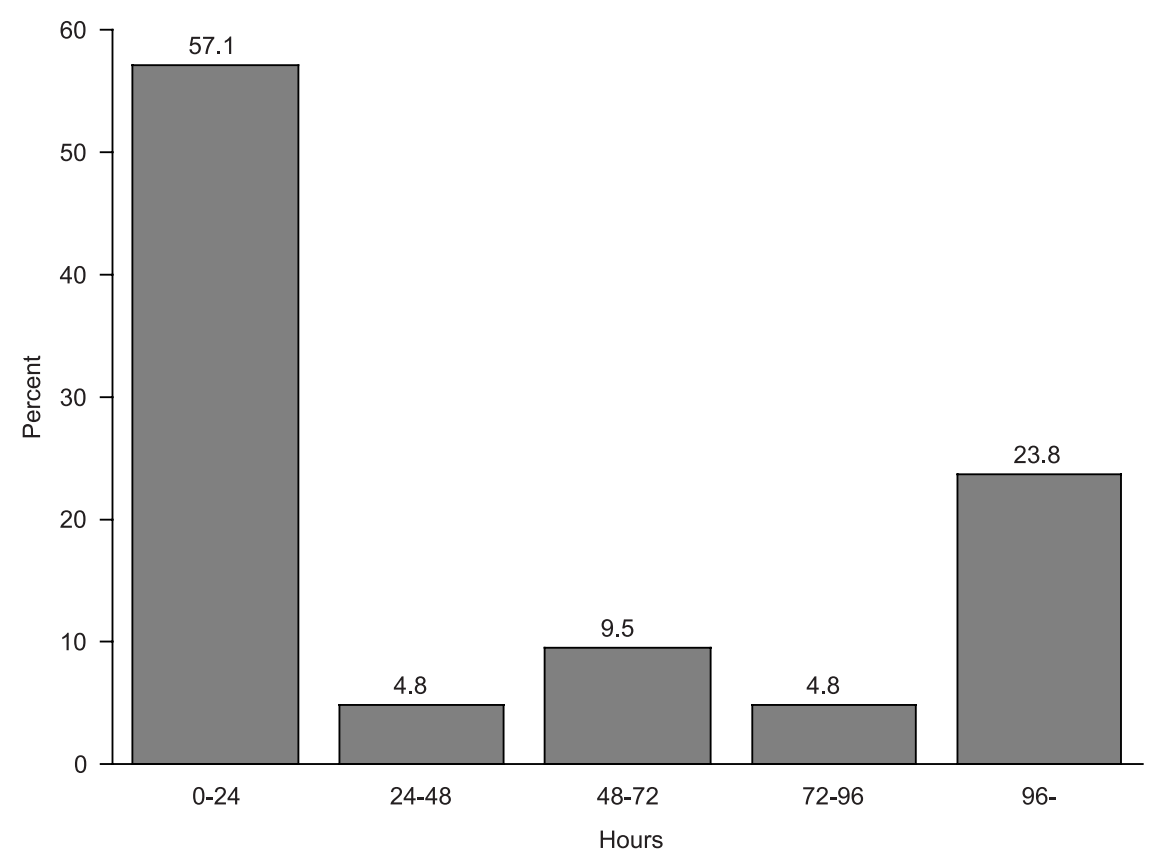

FIG. 2. Time from admittance and start of treatment to craniectomy.

ICP and CPP before and after craniectomy. Mean ICP just before the craniectomy was $36.4 \pm 3.4 \mathrm{~mm} \mathrm{Hg}$, and directly after craniectomy it was $13.1 \pm 2.1 \mathrm{~mm} \mathrm{Hg}(p<$ 0.001 ANOVA, $p<0.001$ post-hoc). This marked reduction of ICP was still significant $72 \mathrm{~h}$ after the procedure ( $p<0.001$ post-hoc). However, there was a slow increase after the first marked decrease, and the ICP leveled out at about $25 \mathrm{~mm} \mathrm{Hg}$.

As demonstrated in Figure 4, there was a non-statistically significant ( $p>0.05$, ANOVA) decrease in MAP during a few hours immediately after the craniectomy.

Follow-up was accomplished in 92 patients. One patient was discharged to his home country, Spain, and was lost to follow-up. The outcome at follow-up is shown in Table 2. In the DC group, favorable outcome (GOS 5-4) was found in $71 \%$; whereas in the whole treatment cohort, favorable outcome was found in $63 \%$. In the NDC group, the corresponding value was $61 \%$. The difference in GOS favorable outcome between the two groups was not statistically significant ( $p>0.05$, Mann-Whitney $U$ test). In both the DC group and the NDC group 14\% were deceased within 3 months of the trauma. If one applies the usual criteria for treatment (non-dilated reacting pupils, GCS of $>3$ ), only $4 \%$ of all patients in the study group died. Four additional patients died during the follow-up time. There was a correlation between the GCS

Table 1. Number, Gender, Age, and GCS at Inclusion

\begin{tabular}{|c|c|c|c|c|c|c|c|c|c|}
\hline & \multicolumn{3}{|c|}{ Craniectomy } & \multicolumn{3}{|c|}{ Non-craniectomy } & \multicolumn{3}{|c|}{$A l l$} \\
\hline & Male & Female & All & Male & Female & All & Male & Female & All \\
\hline Number & 15 & 6 & 21 & 56 & 16 & 72 & 71 & 22 & 93 \\
\hline \multicolumn{10}{|l|}{ Age } \\
\hline Mean & 39.0 & 39.5 & 39.1 & 38.2 & 33.4 & 37.1 & 38.4 & 35.0 & 37.6 \\
\hline Median & 40 & 39.5 & 40 & 33.5 & 29 & 33 & 38 & 31.5 & 35 \\
\hline Range & $15-61$ & $16-60$ & $15-61$ & $16-69$ & $15-58$ & $15-69$ & $15-69$ & $15-60$ & $15-69$ \\
\hline \multicolumn{10}{|l|}{ GCS } \\
\hline Mean & 6.3 & 6.8 & 6.5 & 5.7 & 6.7 & 5.9 & 6.0 & 6.7 & 6.1 \\
\hline Median & 7 & 6.5 & 7 & 6 & 7 & 7 & 6 & 7 & 7 \\
\hline Range & $8-3$ & $8-6$ & $8-3$ & $8-3$ & $7-5$ & $8-3$ & $8-3$ & $8-5$ & $8-3$ \\
\hline
\end{tabular}

GCS, Glasgow Coma Scale. 


\section{OLIVECRONA ET AL.}

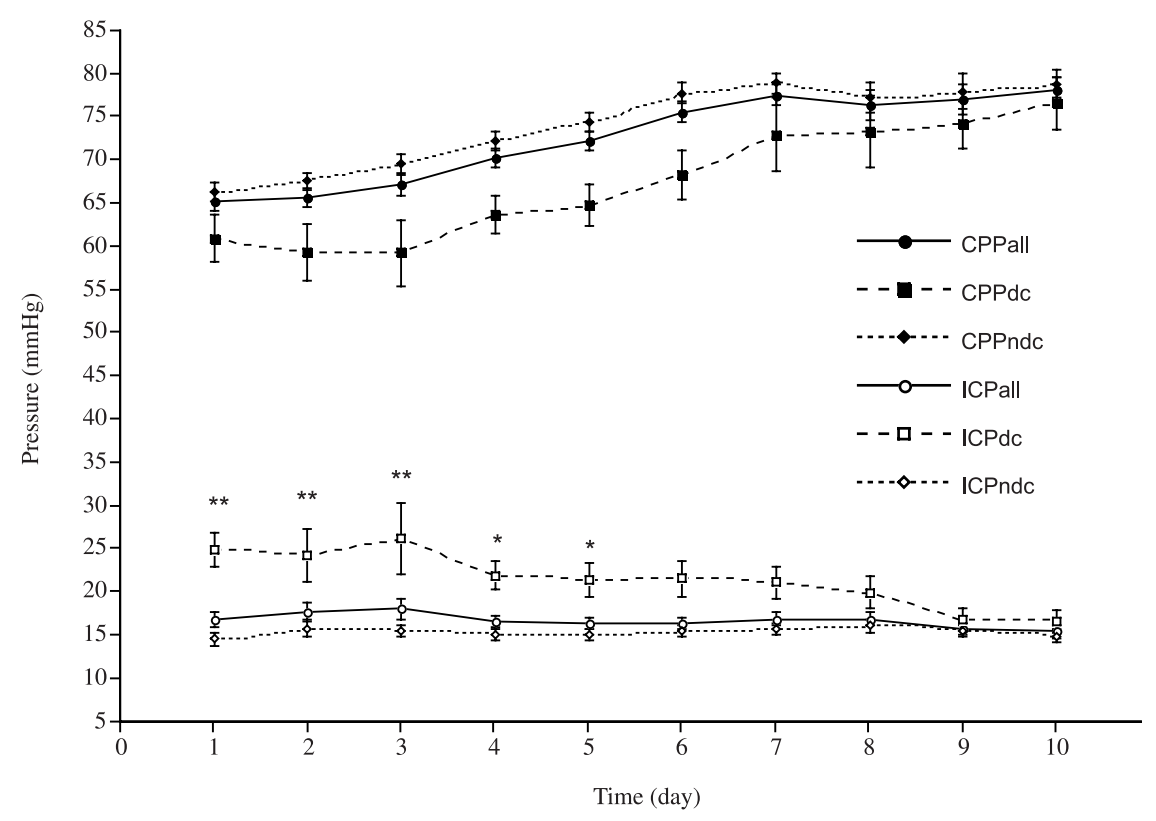

FIG. 3. Daily mean intracranial pressure (ICP) and cerebral perfusion pressure (CPP) during the first 10 days in the total cohort (all), craniectomized group (dc), and non-craniectomized group (ndc). *p $\leq 0.05$ and $* * p \leq 0.01$, analysis of variance (ANOVA) with post-hoc Fisher PLSD, as compared between the dc and ndc groups.

and GOS $(r=0.56, p<0.001)$ as well as between age and $\operatorname{GOS}(r=-0.45, p<0.001)$.

\section{DISCUSSION}

DC is one option in the treatment of raised ICP. The aim with craniectomy in the present study was to reduce
ICP over time and limit it to non-dangerous levels in patients in whom ICP could not be brought under control by other surgical or medical means. We showed that the reduction of ICP achieved by the craniectomy was significant over the first $72 \mathrm{~h}$ following the craniectomy, thus reaching the aim of our intervention. The rapid trend in ICP increase observed just before the craniectomy was clearly interrupted by the procedure. Even though the ul-

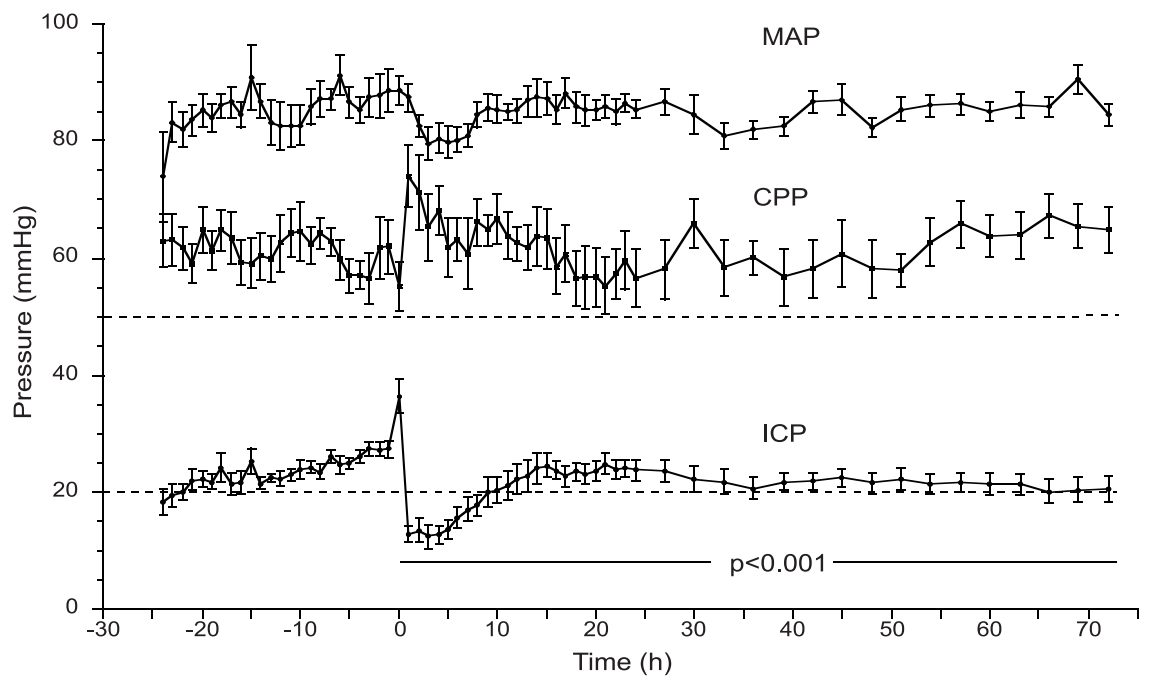

FIG. 4. Mean arterial pressure (MAP), cerebral perfusion pressure (CPP), and intracranial pressure (ICP) trends preceding and after the craniectomy. Analysis of variance (ANOVA) with post-hoc Fisher PLSD. 


\section{DECOMPRESSIVE CRANIECTOMY AND ICP REDUCTION}

Table 2. GOS at Follow-uP

\begin{tabular}{|c|c|c|c|c|c|c|}
\hline \multirow[b]{2}{*}{ GOS } & \multicolumn{2}{|c|}{ Craniectomized $(\mathrm{n}=21)$} & \multicolumn{2}{|c|}{ Non-craniectomized $(\mathrm{n}=71)$} & \multicolumn{2}{|c|}{ All $(\mathrm{n}=92)$} \\
\hline & $\mathrm{n}$ & $\%$ & $\mathrm{n}$ & $\%$ & $\mathrm{n}$ & $\%$ \\
\hline 5 & 9 & 42.9 & 28 & 39.4 & 37 & 40.2 \\
\hline 4 & 6 & 28.6 & 15 & 21.1 & 21 & 22.8 \\
\hline 3 & 2 & 9.6 & 15 & 21.1 & 17 & 18.5 \\
\hline 2 & 1 & 4.8 & 3 & 4.2 & 4 & 4.4 \\
\hline 1 & 3 & 14.3 & 10 & 14.1 & 13 & 14.1 \\
\hline
\end{tabular}

GOS, Glasgow Outcome Scale.

timate goal of $20 \mathrm{~mm} \mathrm{Hg}$ was not accomplished, ICP stabilized at an acceptable level and adequate CPP was not compromised. This goal, of keeping the ICP below 20 $\mathrm{mm} \mathrm{Hg}$, was reached in the NDC group. There were no significant changes in the MAP, either over time, or compared just before and after craniectomy. The other cornerstone of the Lund concept, not allowing CPP to fall below the level of $50 \mathrm{~mm} \mathrm{Hg}$, was met in both subgroups.

In accordance with our findings, Schneider et al. (2002) reported an increase in ICP from $9.8 \mathrm{~mm} \mathrm{Hg} \mathrm{di-}$ rectly after craniectomy to $21.6 \mathrm{~mm} \mathrm{Hg}$ at $12 \mathrm{~h}$ after the procedure. Similar trends were found in two other publications where ICP values over time were provided (Whitfield et al., 2001; Hejazi et al., 2002). The explanation of this increase in ICP after the initial reduction might be related to the drastically changed physiological environment of the brain, possibly leading to transient brain edema formation.

Craniectomy is a major intervention in the physiology and in the natural regulation of blood and cerebrospinal fluid (CSF) dynamics in the brain (Fodstad et al., 1979, 1984; Rinaldi et al., 1990). Yamakami and Yamaura (1993) have shown that cerebral blood flow (CBF) is increased in the decompressed brain within $24 \mathrm{~h}$ after DC. The hyper-perfused area increased during the first week, corresponding to the brain swelling detected on CT scan during the same time (Yamakami and Yamaura, 1993). The mechanism behind this swelling is most probably the loss of resistance in the brain underlying the craniectomy, which would lead to increased transcapillary leakage due to a higher transcapillary hydrostatic pressure gradient. These changes after DC, with edema formation due to the decompression itself, underline the importance of combining the decompression of bone with a large duraplasty. This is to allow for maximal swelling of the brain, avoiding a secondarily raised ICP. The most important factor in avoiding or controlling the secondarily rising ICP is the principle of the Lund concept in which the transcapillary leakage is minimized by reduction of transcapillary pressure-which is achieved through pre- venting high arterial pressure - and by maintaining a normal colloid osmotic pressure using infusion of albumin and packed red blood cells.

Polin et al. (1997) compared ICP at 48-72 h after craniectomy with a control population from the Traumatic Coma Data Bank and found a significantly reduced ICP compared with the ICP of matched controls. We have identified six publications reporting on the change of ICP, comparing ICP values before and after craniectomy used as a step in a protocol-driven treatment (Polin et al., 1997; Kunze et al., 1998; Munch et al., 2000; Whitfield et al., 2001; Kontopoulos et al., 2002; Schneider et al., 2002). Munch et al. (2000) also reported a significant reduction of the shift of the midline structures after craniectomy, noting a reduction in the shift of midline structures from $9.7 \pm 5.4 \mathrm{~mm}$ to $6.2 \pm 5.1 \mathrm{~mm}$. The previous publications applied different intervention limits concerning ICP ranging from 25 to $40 \mathrm{~mm} \mathrm{Hg}$. They all reported a marked decrease in ICP when comparing before and after craniectomy. This was in accordance with our findings. To evaluate the effect of DC, one has to study the results in a formally controlled setting. The procedure must have a distinct place in a protocol-guided treatment program with the intention of achieving a clear goal with the intervention. We showed that DC reduced ICP and interrupted a malignant increase of ICP, and that the lowered ICP was maintained over time in the setting of a protocol-driven treatment.

To achieve this ICP reduction, it appears to be necessary to achieve an adequate volume gain by the craniectomy. Otherwise, brain herniation through a too small craniectomy will result in strangulation of the border vessels of the brain under the craniectomy edges. Unfortunately, not many publications report on the size of the craniectomy, and thus it is difficult to compare results. We have found only one publication reporting on the area and the gained volume by craniectomy in patients with TBI (Munch et al., 2000). This study reported an estimated mean craniectomy area of $67.9 \pm 15.5 \mathrm{~cm}^{2}$ and a volume gain of $92.6 \pm 65.0 \mathrm{~cm}^{3}$. This volume was close 


\section{OLIVECRONA ET AL.}

to the calculated volume in our series. We consider the size and volume gain in our patients to have been large enough, most likely, to provide an unrestricted and adequate brain volume expansion.

Late after craniectomy, a reduced $\mathrm{CBF}$ has been shown not just in the brain underlying the craniectomy but also in the brain contralateral to the craniectomy. These changes seem to normalize after cranioplasty (Yoshida et al., 1996; Winkler et al., 2000). Thus, we recommend that "prophylactic craniectomies" be avoided. One can speculate that these changes in physiology would also indicate the importance of replacing the skull bone or performing a cranioplasty as soon as possible following the craniectomy.

After introducing the Lund concept, it was shown that patients with the combination of severe head injury, lack of carbon dioxide reactivity, and high ICP were able to survive, and ended up having a good outcome (Asgeirsson et al., 1994). We have previously reported our results in adults and in children using this concept, where a mortality rate of about $10 \%$ and a favorable outcome (GOS 5 and 4) in approximately $70 \%$ of the patients treated was demonstrated (Naredi et al., 2001; Wahlstrom et al., 2005). In the present study, these results were confirmed in patients with DC as an additional step in the TBI treatment.

There have been previous reports on results in craniectomized patients, which have included both early and late craniectomies, along with a heterogeneous patient population (including patients with all different ranges of initial GCS). Our study is one of the few studies where craniectomy was performed within a strict protocol. Previously reported ranges of favorable outcome (GOS 5-4) have been 26-66\%, with a mortality of 14.7-52\% (Gaab et al., 1990; Polin et al., 1997; Kunze et al., 1998; Guerra et al., 1999; Meier et al., 2000; Munch et al., 2000; Whitfield et al., 2001; Kontopoulos et al., 2002; Schneider et al., 2002; Albanese et al., 2003; Meier and Grawe, 2003). Thus, our results in severe TBI were at least as good as the best results in the above-mentioned studies. This could be explained by the fact that the craniectomy was a step in an ongoing protocol-guided treatment program and the craniectomy was used to interrupt a malignant increase in ICP.

In the present study, there was no statistically significant difference in GCS between the two groups; however, the 25 and 75 percentil was 6-8 in DC group and 5-7 in NDC group. Thus one could argue that the patients undergoing craniectomy had a lesser brain injury initially than the patients who did not undergo craniectomy. However, the course of ICP development indicated that the initial GCS was not a reliable marker for the development of marked ICP elevations. This study confirmed previous findings that a correlation between initial GCS and GOS, as well as age and GOS exists.

\section{CONCLUSION}

In conclusion, we found that DC, as a step in a protocol-guided treatment program, has a therapeutic role in interrupting malignant ICP increases. However, a randomized prospective trial would be needed to finally prove this. With our approach to these patients, favorable outcome and low mortality could be achieved. We do not recommend prophylactic craniectomy due to the potentially negative effects of the surgery and the need for a later cranioplasty.

\section{ACKNOWLEDGMENTS}

This study was financially supported by grants from the Department of Neurosciences at Umeå University and from Umeå University Hospital general foundation.

\section{REFERENCES}

ALBANESE, J., LEONE, M., ALLIEZ, J.R., et al. (2003). Decompressive craniectomy for severe traumatic brain injury: evaluation of the effects at one year. Crit. Care Med. 31, 2535-2538.

ASGEIRSSON, B., GRANDE, P.O., and NORDSTROM, C.H. (1994). A new therapy of post-trauma brain oedema based on haemodynamic principles for brain volume regulation. Intensive Care Med. 20, 260-267.

CUSHING, H. (1905). The establishment of cerebral hernia as a decompressive measure for inaccessible brain tumours; with the description of intermuscular methods of making the bone defect in temporal and occipital regions. Surg. Gynecol. Obstet. 1, 297-314.

EKER, C., ASGEIRSSON, B., GRANDE, P.O., SCHALEN, W., and NORDSTROM, C.H. (1998). Improved outcome after severe head injury with a new therapy based on principles for brain volume regulation and preserved microcirculation. Crit. Care Med. 26, 1881-1886.

FODSTAD, H., EKSTEDT, J., and FRIDEN, H. (1979). CSF hydrodynamic studies before and after cranioplasty. Acta Neurochir. Suppl. (Wien) 28, 514-518.

FODSTAD, H., LOVE, J.A., EKSTEDT, J., FRIDEN, H., and LILIEQUIST, B. (1984). Effect of cranioplasty on cerebrospinal fluid hydrodynamics in patients with the syndrome of the trephined. Acta Neurochir. (Wien) 70, 21-30.

GAAB, M.R., RITTIERODT, M., LORENZ, M., and HEISSLER, H.E. (1990). Traumatic brain swelling and op- 


\section{DECOMPRESSIVE CRANIECTOMY AND ICP REDUCTION}

erative decompression: a prospective investigation. Acta Neurochir. Suppl. (Wien) 51, 326-328.

GEORGIADIS, D., SCHWARZ, S., ASCHOFF, A., and SCHWAB, S. (2002). Hemicraniectomy and moderate hypothermia in patients with severe ischemic stroke. Stroke 33, $1584-1588$.

GRANDE, P.O., ASGEIRSSON, B., and NORDSTROM, C.H. (1997). Physiologic principles for volume regulation of a tissue enclosed in a rigid shell with application to the injured brain. J. Trauma 42, Suppl, S23-S31.

GRANDE, P.O., ASGEIRSSON, B., and NORDSTROM, C.H. (2002). Volume-targeted therapy of increased intracranial pressure: the Lund concept unifies surgical and non-surgical treatments. Acta Anaesthesiol. Scand. 46, 929-941.

GUERRA, W.K., GAAB, M.R., DIETZ, H., MUELLER, J.U., PIEK, J., and FRITSCH, M.J. (1999). Surgical decompression for traumatic brain swelling: indications and results. J. Neurosurg. 90, 187-196.

HEJAZI, N., WITZMANN, A., and FAE, P. (2002). Unilateral decompressive craniectomy for children with severe brain injury. Report of seven cases and review of the relevant literature. Eur. J. Pediatr. 161, 99-104.

KOCHER, E.T. (1901). Die Therapie des Hirndruckes, in: Specielle Pathologie und Therapie. H. Nothnagel (ed), Alfred Hölder: Wien, pps. 255-266.

KONTOPOULOS, V., FOROGLOU, N., PATSALAS, J., et al. (2002). Decompressive craniectomy for the management of patients with refractory hypertension: should it be reconsidered? Acta Neurochir. (Wien) 144, 791-796.

KUNZE, E., MEIXENSBERGER, J., JANKA, M., SORENSEN, N., and ROOSEN, K. (1998). Decompressive craniectomy in patients with uncontrollable intracranial hypertension. Acta Neurochir. Suppl. 71, 16-18.

MALM, J., BERGENHEIM, A.T., ENBLAD P., et al. (2006). The Swedish Malignant Middle Cerebral Artery Infartion Study: long-term results from a prospective study of hemicraniectomy combind with standardized neurointensive care. Acta Neurol. Scand. 113, 25-30.

MEIER, U., and GRAWE, A. (2003). The importance of decompressive craniectomy for the management of severe head injuries. Acta Neurochir. Suppl. 86, 367-371.

MEIER, U., ZEILINGER, F.S., and HENZKA, O. (2000). The use of decompressive craniectomy for the management of severe head injuries. Acta Neurochir. Suppl. 76, 475-478.

MUNCH, E., HORN, P., SCHURER, L., PIEPGRAS, A., PAUL, T., and SCHMIEDEK, P. (2000). Management of severe traumatic brain injury by decompressive craniectomy. Neurosurgery 47, 315-322.

NAREDI, S., EDEN, E., ZALL, S., STEPHENSEN, H., and RYDENHAG, B. (1998). A standardized neurosurgical neurointensive therapy directed toward vasogenic edema after severe traumatic brain injury: clinical results. Intensive Care Med. 24, 446-451.
NAREDI, S., OLIVECRONA, M., LINDGREN, C., OSTLUND, A.L., GRANDE, P.O., and KOSKINEN, L.O. (2001). An outcome study of severe traumatic head injury using the "Lund therapy" with low-dose prostacyclin. Acta Anaesthesiol. Scand. 45, 402-406.

POLIN, R.S., SHAFFREY, M.E., BOGAEV, C.A., et al. (1997). Decompressive bifrontal craniectomy in the treatment of severe refractory posttraumatic cerebral edema. Neurosurgery $41,84-92$.

RINALDI, A., MANGIOLA, A., ANILE, C., MAIRA, G., AMANTE, P., and FERRARESI, A. (1990). Hemodynamic effects of decompressive craniectomy in cold induced brain oedema. Acta Neurochir. Suppl. (Wien) 51, 394-396.

SCHNEIDER, G.H., BARDT, T., LANKSCH, W.R., and UNTERBERG, A. (2002). Decompressive craniectomy following traumatic brain injury: ICP, CPP and neurological outcome. Acta Neurochir. Suppl. 81, 77-79.

SCHWAB, S., STEINER, T., ASCHOFF, A., et al. (1998). Early hemicraniectomy in patients with complete middle cerebral artery infarction. Stroke 29, 1888-1893.

WAHLSTROM, M.R., OLIVECRONA, M., KOSKINEN, L.O., RYDENHAG, B., and NAREDI, S. (2005). Severe traumatic brain injury in pediatric patients: treatment and outcome using an intracranial pressure targeted therapy-the Lund concept. Intensive Care Med. 31, 832-839.

WHITFIELD, P.C., PATEL, H., HUTCHINSON, P.J., et al. (2001). Bifrontal decompressive craniectomy in the management of posttraumatic intracranial hypertension. Br. J. Neurosurg. 15, 500-507.

WINKLER, P.A., STUMMER, W., LINKE, R., KRISHNAN, K.G., and TATSCH, K. (2000). Influence of cranioplasty on postural blood flow regulation, cerebrovascular reserve capacity, and cerebral glucose metabolism. J. Neurosurg. 93, 53-61.

YAMAKAMI, I., and YAMAURA, A. (1993). Effects of decompressive craniectomy on regional cerebral blood flow in severe head trauma patients. Neurol. Med. Chir. (Tokyo) 33, 616-620.

YOSHIDA, K., FURUSE, M., IZAWA, A., IIZIMA, N., KUCHIWAKI, H., and INAO, S. (1996). Dynamics of cerebral blood flow and metabolism in patients with cranioplasty as evaluated by ${ }^{133} \mathrm{Xe} \mathrm{CT}$ and ${ }^{31} \mathrm{P}$ magnetic resonance spectroscopy. J. Neurol. Neurosurg. Psychiatry 61, $166-171$.

\section{Address reprint requests to: Magnus Olivecrona, M.D. Department of Neurosurgery University Hospital SE 90185 Umeå, Sweden}

E-mail: magnus.olivecrona@vll.se 